\title{
Spatial Distribution and Accessibility to Public Sector Tertiary Care Teaching General Hospitals: Tale of Two Khyber Pakhtunkhwa Districts - Peshawar and Abbottabad
}

\author{
Masood Ali Shaikh1 and Naeem Abbas Malik²
}

\begin{abstract}
Effective utilisation of healthcare facilities is determined by spatial and non-spatial factors, including their spatial distribution and physical accessibility. Public sector plays an important role in the provision of healthcare services for improving health in developing countries like Pakistan. We analysed the spatial distribution and accessibility to public sector tertiary care teaching and general hospitals in the Khyber Pakhtunkhwa (KPK) districts of Abbottabad and Peshawar. Two such hospitals in Abbottabad and three in Peshawar were selected for the analyses. Out of the 44 union councils (UCs) in Abbottabad, there are 23 and 15 UCS that are partially or fully accessible within the 12-kilometer buffer and the service area around the hospitals, respectively. Out of the 93 UCs in Peshawar, there are 79 and 77 UCs that are partially or fully accessible, respectively, within the 12-kilometer buffer and service area around the hospitals.
\end{abstract}

Key Words: Hospitals, Accessibility, GIS, Pakistan.

tilisation of healthcare services is impacted by several spatial and non-spatial factors.1,2 Spatial factors influencing spatial accessibility involve ease of physical accessibility to the health facilities, in addition to travel time and monetary cost associated with such travel for seeking preventive and curative healthcare services. Spatial distribution and physical accessibility of healthcare facilities also determine effective utilisation of healthcare services for reducing disease morbidity and mortality burden. ${ }^{3}$

The ability to pay for healthcare services, especially specialised and tertiary care services, poses significant financial burden on people from lower and middleincome strata in developing countries. Public sector plays a pivotal part in providing healthcare services and reducing disease burden for the populations. ${ }^{3}$ In Pakistan, the use of public sector tertiary care hospitals linked with medical colleges and universities, is deemed as trusted, affordable, and quality source of specialised curative healthcare services.

There is only one study on spatial accessibility to public sector tertiary care general and teaching hospitals in Pakistan, which was conducted in the city of Karachi, using geographic information system and network analysis. ${ }^{4}$ Peshawar and Abbottabad districts are home

\footnotetext{
1 Independent Consultant, Block No. 7, Gulshan-e-Iqbal, Karachi, Pakistan

2 Institute of Geo-Information and Earth Observation, PMAS Arid Agriculture University, Rawalpindi, Pakistan

Correspondence: Dr. Masood Ali Shaikh, Independent Consultant, Block No. 7, Gulshan-e-Iqbal, Karachi, Pakistan E-mail:masoodali1@yahoo.com

Received: June 13, 2018; Accepted: September 18, 2018
}

to the two oldest public sector medical colleges in the Khyber Pakhtunkhwa (KPK) province in the northwestern part of Pakistan. In terms of population size, Peshawar is the largest district in the KPK; while Abbottabad district is the ninth largest district in KPK, based on the 2017 population census. ${ }^{5}$

Straight line (from the point location of hospitals to the radius of specified distance), and road network-based (specified distance traversed on roads) distances are two common methods of modelling spatial accessibility to healthcare services. 1,4

The objectives of this study were to model the spatial accessibility to public sector teaching tertiary care general hospitals in the two KPK districts, Peshawar and Abbottabad, using buffers and road network-based service areas.

Two public sector teaching general hospitals in Abbottabad, i.e. Ayub Teaching Hospital and District Headquarters (DHQs) Hospital; three public sector teaching general hospitals in Peshawar, i.e. Hayatabad Medical Complex, Khyber Teaching Hospital, and Lady Reading Hospital, were selected for spatial distribution and accessibility analyses. From Google Earth, the latitude and longitude ( $X$ and $Y$ ) coordinations for all five hospitals were obtained. The vector road network files for the districts of Abbottabad and Peshawar were downloaded from the Open Street Map. 6 Geographic Information Systems (GIS) analyses and mapping were done with the ArcGIS programme, version 10.5. ArcGIS is a software for working with geographic data, and is used for creating and analysing mapped information. The road network file for Pakistan was clipped (extracted) using the Abbottabad and Peshawar districts boundary shape files. All three GIS files, i.e. boundary 
files, hospital location point files, and road network files for both districts were projected in Universal Transverse Mercator (UTM) coordinate system. The UTM projection is one of the projections which is based on 2dimensional Cartesian coordinate system that displays the 3-dimensional earth on a 2-dimensional paper.

The Figures 1 and 2 show the Union Councils (UCs), road network, and point locations of hospitals in Abbottabad and Peshawar, respectively. The two hospitals in Abbottabad are located in the north-central part of the district; while in Peshawar, the three hospitals are located in the east-central and central parts of the district. Buffers around point locations of hospitals were created, based on straight line distances of 3,6, 9, and 12 kilometers. The Network Analyst Extension of the GIS analysis software ArcGIS 10.5 was used for creating spatial accessibility analysis. Based on road network, service areas of $3,6,9$ and 12 kilometers were created. Using Abbottabad and Peshawar road network shape files, road networks for each district were built in the Network Analyst Extension of ArcGIS programme. Service areas were created with the ArcGIS's 'Network Analyst' Extension, using 'Any Vertex' connectivity policy, while ignoring the elevation and direction of travel permitted on the roads in terms of one-way or two-way

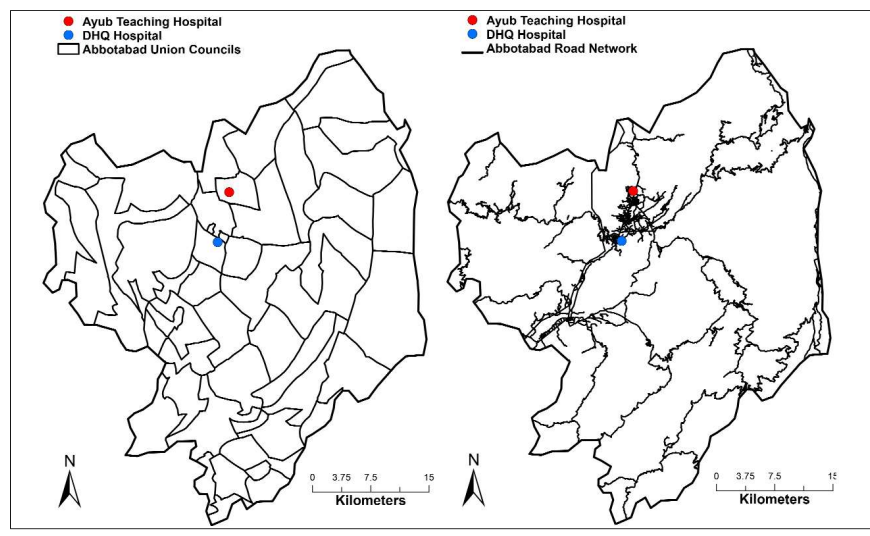

Figure 1: Abbottabad union councils (left map), road network (right map) and public sector, tertiary care, teaching, general hospitals.

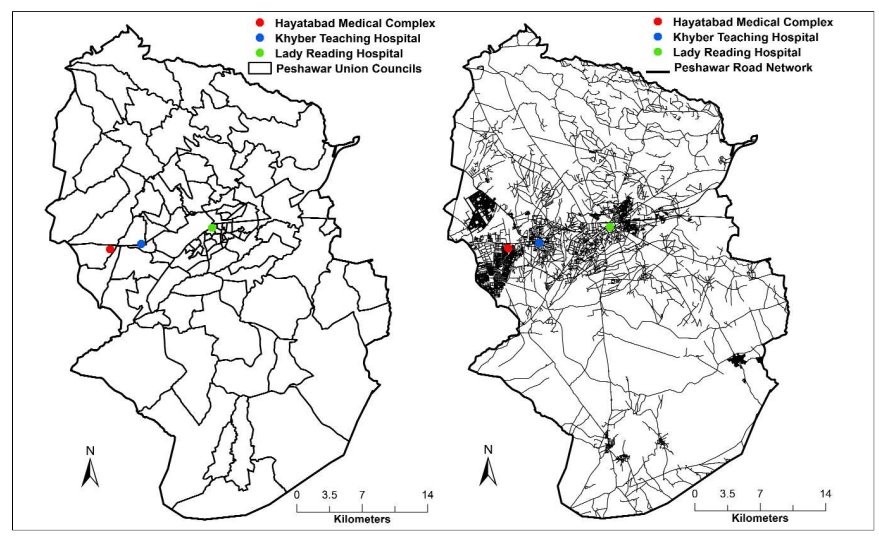

Figure 2: Peshawar union councils (left map), road network (right map) and public sector tertiary care teaching general hospitals. traffic flow. Both buffers as well as service areas for hospitals in each district were dissolved to portray an overall accessibility to any of these hospitals in each district.

Figures 3 and 4 show the 3, 6, 9, and 12 kilometersbased service areas around the hospitals on the left maps and straight line distance based $3,6,9$, and 12 kilometers-based buffers around the hospitals on the right map, for Abbottabad and Peshawar. Collectively, all maps show the UCs that are either partially or completely covered/accessible by the service area and buffers around the hospitals. Out of the 44 UCs in Abbottabad, there are 23 and 15 UCs that are either partially or fully accessible within the 12-kilometer buffer and the service area, respectively. While out of the 93 UCs in Peshawar, there are 79 and 77 UCs that are either partially or fully accessible within the 12-kilometer buffer and service area, respectively.

Results show that these hospitals are located in the north-central part of the district in Abbottabad, and in the east-central and central part of Peshawar. In Peshawar, only 14 and 16 UCs are not fully or partially accessible within the 12-kilometer buffer and service areas, respectively. As information on population size by UCs is not available, it is not possible to determine how many

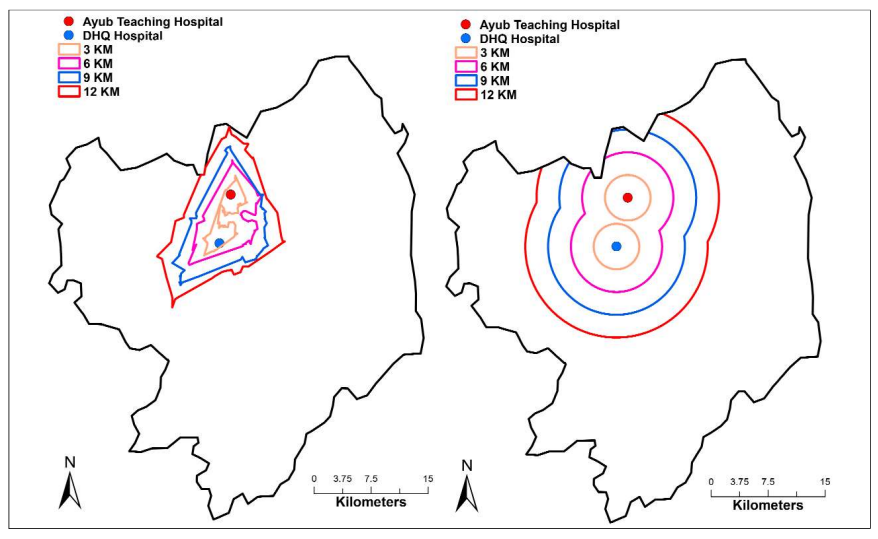

Figure 3: Service areas (left map) and buffers (right map) around public sector, tertiary care, teaching, general hospitals in Abbottabad.
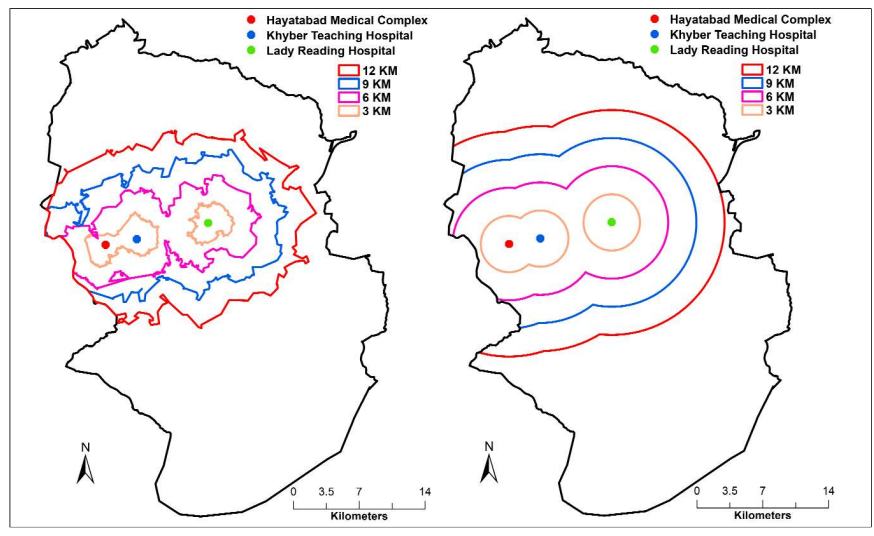

Figure 4: Service areas (left map) and buffers (right map) around public sector tertiary care teaching general hospitals in Peshawar. 
people have geographical accessibility to the hospitals. Availability of comprehensive GIS and population data at the UCs level would facilitate more informed health policy-making in the country. Further studies are needed on this subject to fully understand the access and availability of specialised healthcare facilities in the whole country.

\section{REFERENCES}

1. Seymour JW, Polsky DE, Brown EJ, Barbu CM, Grande D. The role of community health centers in reducing racial disparities in spatial access to primary care. J Prim Care Community Health 2017; 8:147-52.

2. Biswas RK, Kabir E. Influence of distance between residence and health facilities on non-communicable diseases: An assessment over hypertension and diabetes in Bangladesh. PLoS One 2017; 12:e0177027.

3. Goudge J, Gilson L, Russell S, Gumede T, Mills A. Affordability, availability and acceptability barriers to healthcare for the chronically ill: Longitudinal case studies from South Africa. BMC Health Serv Res 2009; 9:75.

4. Shaikh MA, Ali MS. Spatial distribution and accessibility to public sector tertiary care teaching hospitals in Karachi: A geographic information systems application. J Pak Med Assoc 2016; 66:889-92.

5. Districts of Khyber Pakhtunkhwa. https://en.wikipedia.org/wiki/ Districts_of_Khyber_Pakhtunkhwa [cited 2018 April 11].

6. OpenStreetMap. https://www.openstreetmap.org/\#map=11/31.9402/ 36.0750 [cited 2018 March 29]. 